\title{
Role of nuclear physics in oscillations of magnetars
}

\author{
Rana Nandi ${ }^{1}$, Prasanta Char ${ }^{2}$, Debarati Chatterjee ${ }^{3,}$, and Debades Bandyopadhyay ${ }^{2}$ \\ ${ }^{1}$ Frankfurt Institute for Advanced Studies, \\ Ruth Moufang Strasse 1, 60438 Frankfurt am Main, Germany \\ ${ }^{2}$ Astroparticle Physics and Cosmology Division and Centre for Astroparticle Physics, \\ Saha Institute of Nuclear Physics, HBNI, \\ 1/AF Bidhannagar, Kolkata, 700064, India and \\ ${ }^{3}$ Laboratoire de Physique Corpusculaire, ENSICAEN, \\ 5 Boulevard Maréchal Juin, F-14050 Caen, France
}




\begin{abstract}
Strong magnetic fields have important effects on the crustal properties of magnetars. Here we study the magneto-elastic oscillations of magnetars taking into consideration the effect of strong magnetic fields on the crustal composition (magnetised crust). We calculate global magneto-elastic (GME) modes as well as modes confined to the crust (CME) only. The ideal magnetohydrodynamics is adopted for the calculation of magneto-elastic oscillations of magnetars with dipole magnetic fields. The perturbation equations obtained in general relativity using Cowling approximation are exploited here for the study of magneto-elastic oscillations. Furthermore, deformations due to magnetic fields and rotations are neglected in the construction of equilibrium models for magnetars. The composition of the crust directly affects its shear modulus which we calculate using three different nucleon-nucleon interactions: SLy4, SkM and Sk272. The shear modulus of the crust is found to be enhanced in strong magnetic fields $\geq 10^{17} \mathrm{G}$ for all those Skyrme interactions. It is noted that the shear modulus of the crust for the SLy4 interaction is much higher than those of the SkM and Sk272 interactions in presence of magnetic fields or not. Though we do not find any appreciable change in frequencies of fundamental GME and CME modes with and without magnetised crusts, frequencies of first overtones of CME modes are significantly affected in strong magnetic fields $\geq 10^{17} \mathrm{G}$. However, this feature is not observed in frequencies of first overtones of GME modes. As in earlier studies, it is also noted that the effects of crusts on frequencies of both types of maneto-elastic modes disappear when the magnetic field reaches the critical field $\left(B>4 \times 10^{15}\right.$ G). Frequencies of GME and CME modes calculated with magnetised crusts based on all three nucleon-nucleon interactions, stellar models and magnetic fields, are compared with frequencies of observed quasi-periodic oscillations (QPOS) in SGR 1806-20 and SGR1900+14. As in earlier studies, this comparison indicates that GME modes are essential to explain all the frequencies as CME modes can explain only the higher frequencies.
\end{abstract}

PACS numbers: 97.60.Jd, 47.75.+f, 95.30.Sf 


\section{INTRODUCTION}

Soft gamma repeaters (SGRs) are characterised by their sporadic and short bursts of soft gamma rays. Luminosities in these bursts could reach as high as $\sim 10^{41}$ ergs s$^{-1}$. There are about 14 SGRs known observationally [1]. Evidence of stronger emissions of gamma rays from SGRs was observed in several cases. These events are known as giant flares in which luminosities are $\sim 10^{44}-10^{46} \mathrm{ergs}^{-1}$. So far three cases of giant flares were reported and those are SGR 0526-66, SGR 1900+14 and SGR 1806-20 [2 6]. In giant flares, the early part of the spectrum was dominated by a hard flash of shorter duration followed by a softer decaying tail of a few hundreds of seconds.

SGRs are very good candidates for magnetars which are neutron stars with very high surface magnetic fields $\sim 10^{15} \mathrm{G}[7[9]$. Giant flares might be caused by the evolving magnetic field and its stress on the crust of magnetars. It was argued that starquakes associated with giant flares could excite Global Seismic Oscillations (GSOs)[8]. Torsional shear modes of magnetars with lower excitation energies would be easily excited. In this case, oscillations are restored by the Coulomb forces of crustal ions. Furthermore, the torsional shear modes have longer damping times. These findings implied that QPOs might be shear modes of magnetars [8]. Quasi-periodic oscillations were found in the decaying tail of giant flares of SGR 1806-20 and SGR 1900+14. Detected frequencies for SGR 1806-20 are 18, 26, 29, 92.5, 150, 626.5 and $1837 \mathrm{~Hz}$ [4, 10, 11], whereas for SGR 1900+14 detected frequencies are 28, 53.5, 84 and $155 \mathrm{~Hz}$ [12]. Huppenkothen et al [13 15] recently analyzed short bursts of some SGRs and found QPOs with frequencies 93 and $127 \mathrm{~Hz}$ in SGR J1550-5418 [13] and with $57 \mathrm{~Hz}$ in SGR 1806-20 [14].

It was noted from earlier theoretical models of QPOs that the observed frequencies, in particular higher frequencies, could be explained reasonably well using pure shear modes as well as CME modes [5, 8, 16 21]. On the other hand, lower frequencies of observed QPOs might be connected to Alfvén modes of the fluid core. This makes the study of the oscillations of magnetar crusts more difficult. There were attempts to explain frequencies of QPOs using Alfvén oscillations of the fluid core without considering a crust [19, 22 24]. Levin [22, 25] first pointed out that the strong magnetic fields of magnetars should couple the Alfvén oscillations of fluid core with the oscillations in the solid crust. After that many authors studied the problem in detail [26-30]. The magnetohydrodynamics (MHD) coupling 
between the crust and core causes pure crustal modes to decay by emitting Alfvén waves into the core. It was argued that CME modes might appear in GSOs and explain frequencies of observed QPOs for not very strong magnetic fields despite all these complex problems 31]. But global modes are expected to couple to the Alfvén continuum in the core, and leads to the damping of the modes. Simulations to simplified models show [22, 26] that CME oscillations are efficiently damped in the Alfvén continuum as the crust reacts to the motion of the core. Consequently long-lived QPOs can be generated at special points of the continuum (turning points and edges of continuum).

Several groups also studied the effect of neutron superfluidity and/or proton superconductivity of the crust and/or core on the calculated frequencies of magnetars [32 34]. It was noted that neutron superfluidity enhanced fundamental frequencies of magneto-elastic oscillations. On the other hand, it was argued that proton superconductivity could be destroyed in magnetic fields $>5 \times 10^{16} \mathrm{G}[35]$.

Nuclear physics of crusts plays an important role on the magneto-elastic modes of magnetars. In particular, the effects of the nuclear symmetry energy on the CME frequencies were investigated recently [21]. It may be worth noting here that CME mode frequencies are sensitive to the shear modulus of neutron star crusts. Furthermore, the shear modulus strongly depends on the composition of neutron star crusts. In earlier studies of magnetoelastic modes the effect of magnetic field on the composition of the crust was not considered. Surface magnetic fields as large as $\sim 10^{15} \mathrm{G}$ have been reported in magnetars. Further, indirect estimates using the scalar virial theorem does not exclude internal magnetic fields up to $10^{18}$. Such large magnetic fields in magnetars may influence the ground state properties of neutron star crusts. Recently, we have investigated the influence of Landau quantisation of electrons on the compositions and equations of state (EoS) of outer and inner crusts and obtained appreciable changes in those properties when the magnetic field is very strong $\left(B \geq 10^{17} G\right)$ [36, 37]. This, in turn, might influence the shear modulus of crusts and thereby magneto-elastic frequencies of magnetars. This motivates us to study these mode oscillations of magnetars using magnetised crusts. We define the crust to be magnetised (non-magnetised) when the effect of magnetic field on the crustal composition is considered (not considered).

We organise the paper in the following way. We describe models for calculating oscillation modes, shear modulus and compositions and EoS of magnetised crusts in Sec. II. Results of 
this calculation are discussed in Sec. III. Section IV gives the summary and conclusions.

\section{FORMALISM}

QPOs were investigated in Newtonian gravity [8, 16, 38, 39] as well as general relativity [17, 19, 31, 40, 41] with and without magnetic fields. In many of those calculations, the magnetised crust was decoupled from the fluid core. But the magnetic field strongly couples the crust to the core and we need to calculate magneto-elastic modes.

Here we first study the effects of magnetised crusts on the magneto-elastic modes confined to the crust (CME) only, by considering a free slip between the crust and the core. Next, we calculate the global magneto-elastic (GME) modes where coupling between the crust and the core has been considered. Mode frequencies are calculated following the model of Refs. [17, 41, 42]. The spherically symmetric general relativistic model of Sotani et al. [42] adopted in this calculation is a simplified one compared with the state-of-the-art general relativistic magnetohydrodynamical (MHD) model [29]. Furthermore, we do not consider the coupling to the Alfvén continuum within the framework of this study, as the aim of this work primarily is to investigate the influence of magnetised crusts on QPOs.

It is well known that a strong magnetic field breaks the spherical symmetry of a neutron star due to anisotropy of the energy momentum tensor [43]. Hence the isotropic TolmanOppenheimer-Volkoff (TOV) equations are no longer applicable for computing the massradius relations for polar magnetic fields $\sim 10^{17} \mathrm{G}$.

Ideally for large magnetic fields, one must then calculate the neutron star structure using the anisotropic stress-energy tensor and solving equations for hydrostatic equilibrium [44]. Although this approximation is reasonable for magnetic fields $<10^{17} \mathrm{G}$, the deviations from spherical symmetry become non-negligible for higher fields. However, the aim of this work is to study the relative changes in the mode frequencies due to magnetic fields. For this reason, we neglect the deformation of the neutron star and assume it to be spherically symmetric. The metric used to determine equilibrium stellar models has the form,

$$
d s^{2}=-e^{2 \Phi} d t^{2}+e^{2 \Lambda} d r^{2}+r^{2}\left(d \theta^{2}+\sin ^{2} \theta d \phi^{2}\right) .
$$

The equilibrium models are obtained by solving the Tolman-Oppenheimer-Volkoff (TOV) equation with a perfect fluid EoS. 
Here we consider an axisymmetric poloidal magnetic field generated by four current $J_{\mu}=\left(0,0,0, J_{\Phi}\right)$ and expand the four-potential into vector spherical harmonics as $A_{\mu}=$ $a_{\ell_{m}}(r) \sin \theta \partial_{\theta} P_{\ell_{m}}(\cos \theta)$.

The perturbed equations are obtained by linearising the equations of motion of the fluid and the magnetic induction equation [17, 41]. Torsional modes are incompressible and do not result in any appreciable density perturbation in equilibrium stars. Consequently, one may adopt the relativistic Cowling approximation and neglect metric perturbations $\delta g_{\mu \nu}=0$ [45]. We consider axial type perturbation in the four velocity and the relevant perturbed matter quantity is the $\phi$-component of the perturbed four velocity $\partial u^{\phi}[17]$

$$
\partial u^{\phi}=e^{-\Phi} \partial_{t} \mathcal{Y}(t, r) \frac{1}{\sin \theta} \partial_{\theta} P_{l}(\cos \theta),
$$

where $\partial_{t}$ and $\partial_{\theta}$ correspond to partial derivatives with respect to time and $\theta$, respectively, $P_{l}(\cos \theta)$ is the Legendre polynomial of order $l$ and $\mathcal{Y}(t, r)$ is the angular displacement of the matter. It is to be noted that the radial and angular variations of azimuthal displacement of stellar matter lead to shears of the crystal lattice in neutron star crusts which are described by the shear tensor $S_{\mu \nu}$ [40]. Further, the shear stress tensor is given by $T_{\mu \nu}=-2 \mu S_{\mu \nu}$, where $\mu$ is the isotropic shear modulus. The linearised equations of motion includes the contribution of $\delta T_{\mu \nu}$ [17].

Assuming a harmonic time dependence for $\mathcal{Y}(t, r)=e^{i \omega t} \mathcal{Y}(r)$ and neglecting $\ell \pm 2$ terms, one obtains the eigenvalue equation for the mode frequency [17]

$$
\begin{aligned}
& {\left[\mu+\left(1+2 \lambda_{1}\right) \frac{a_{1}{ }^{2}}{\pi r^{4}}\right] } \mathcal{Y}^{\prime \prime}+\left\{\left(\frac{4}{r}+\Phi^{\prime}-\Lambda^{\prime}\right) \mu\right. \\
&+\left.\mu^{\prime}+\left(1+2 \lambda_{1}\right) \frac{a_{1}}{\pi r^{4}}\left[\left(\Phi^{\prime}-\Lambda^{\prime}\right) a_{1}+2 a_{1}{ }^{\prime}\right]\right\} \mathcal{Y}^{\prime} \\
&+\left\{\left[\left(\epsilon+p+\left(1+2 \lambda_{1}\right) \frac{a_{1}{ }^{2}}{\pi r^{4}}\right) e^{2 \Lambda}-\frac{\lambda_{1} a_{1}{ }^{\prime 2}}{2 \pi r^{2}}\right] \omega^{2} e^{-2 \Phi}\right. \\
&-(\lambda-2)\left(\frac{\mu e^{2 \Lambda}}{r^{2}}-\frac{\lambda_{1} a_{1}{ }^{2}}{2 \pi r^{4}}\right) \\
&\left.+\frac{\left(2+5 \lambda_{1}\right) a_{1}}{2 \pi r^{4}}\left[\left(\Phi^{\prime}-\Lambda^{\prime}\right) a_{1}{ }^{\prime}+a_{1}{ }^{\prime \prime}\right]\right\} \mathcal{Y}=0,
\end{aligned}
$$

where $\lambda=\ell(\ell+1)$ and $\lambda_{1}=-\ell(\ell+1) /(2 \ell-1)(2 \ell+3)$. Equation (3) reduces to the nonmagnetic case when we put $a_{1}=0$ [17]. Sotani et al. [20] showed that the $\ell \pm 2$ truncation 
worked well for oscillations confined to the crust only. The eigenvalue equation for modes confined to the crust was solved using a two dimensional numerical method where $\ell \pm 2$ terms were not truncated [20]. It was demonstrated that results were unaffected whether $\ell \pm 2$ terms were truncated or not. With suitable choice of new variables, Eq.(3) results in a system of first order ordinary differential equations [17]. For magnerto-elastic modes confined to the crust, we impose a zero traction boundary condition at the interface between the core and the crust as well as the zero torque condition at the surface [17, 31]. These conditions imply $\mathcal{Y}^{\prime}=0$ at the surface $(r=R)$ of the star and the interface $\left(r=R_{c}\right)$ of the crust and core. For the GME modes the boundary condition at the surface is the same as CME modes [17, 29]. The other boundary condition is the regularity at the center $\left(\mathcal{Y} \sim r^{\ell-1}\right)$. Finally, we estimate eigenfrequencies by solving two first order differential equations.

The knowledge of the shear modulus of magnetised crusts is an important input in the eigenvalue equation [Eq.(3)] for the CME mode calculation. Here we adopt the following expression of the shear modulus at zero temperature [46, 47]

$$
\mu=0.1194 \frac{n_{i}(Z e)^{2}}{a}
$$

where $a=\left[3 /\left(4 \pi n_{i}\right)\right]^{1 / 3}, Z$ is the atomic number of a nucleus and $n_{i}$ is the ion density. This form of the shear modulus was obtained by assuming a bcc lattice and performing directional averages [48]. Further the dependence of the shear modulus on temperature was also investigated with the Monte Carlo sampling technique by Strohmayer et al. [47]. The composition and equation of state of neutron star crusts are essential ingredients for the calculation of the shear modulus as it is evident from Eq.(41).

Now we describe the ground state properties in outer and inner crusts in the presence of strong magnetic fields. The outer crust is composed of nuclei immersed in a uniform background of a non-interacting electron gas. Neutrons start coming out of nuclei when the neutron drip point is reached. This is the beginning of the inner crust where nuclei are placed both in free neutrons as well as electrons. To minimize the Coulomb energy nuclei are arranged in a bcc lattice in neutron star crusts [49].

The ground state properties of matter of the inner crust is described using the ThomasFermi model [36]. The spherical cell that contains neutrons and protons does not define a nucleus. We adopt the procedure of Bonche, Levit and Vautherin to subtract the free 
neutron gas of the cell and obtain the nucleus $[50,52]$.

For neutron star crusts in strongly quantising magnetic fields we showed earlier that the Landau quantisation of electrons strongly influenced ground state properties of neutron star crusts in strong magnetic fields $\sim 10^{17} \mathrm{G}[36$, 37]. Energy and number densities of electrons are affected by the phase space modifications due to Landau quantisation of electrons. It is to be noted that protons are only influenced by magnetic fields through the charge neutrality condition.

\section{RESULTS AND DISCUSSIONS}

We already investigated the composition and EoS of ground state matter in neutron star crusts in strong magnetic fields [36, 37]. We noted that the electron number density in the outer crust was enhanced compared with the field free case when a few Landau levels were populated for magnetic fields $>4.414 \times 10^{16} \mathrm{G}$ [36]. It was observed that this enhancement grew stronger when only the zeroth Landau level was populated at a magnetic field strength of $4.414 \times 10^{17} \mathrm{G}$. Consequently, we found modifications in the sequence of equilibrium nuclei which was obtained by minimising the Gibbs free energy per nucleon. It was noted that some new nuclei such as ${ }_{38}^{88} \mathrm{Sr}$ and ${ }_{46}^{128} \mathrm{Pd}$ appeared and some nuclei such as ${ }^{66} \mathrm{Ni}$ and ${ }^{78} \mathrm{Ni}$ disappeared in a magnetic field of $B=4.414 \times 10^{16} \mathrm{G}[36]$ when we compared this with the zero field case. It was further observed that the neutron drip point was shifted to higher density in presence of a strong magnetic field with respect to the field free case [36]. We also performed the calculation of the inner crust using the SLy4 and SkM nucleon-nucleon interactions [37]. In this case too, we calculated the equilibrium nucleus at each density point. Like the outer crust in strong magnetic fields, the electron number density was enhanced due to the electron population in the zero Landau level for magnetic fields $\geq 10^{17}$ $\mathrm{G}$ which , in turn, led to a large proton fraction because of charge neutrality. For magnetic fields $>10^{17} \mathrm{G}$, equilibrium nuclei with larger mass and atomic numbers were found to exist in the crust [37]. The free energy per nucleon of the nuclear system was reduced in magnetic fields compared with the corresponding case without a magnetic field. Furthermore, it was noted that higher symmetry energy in the sub-saturation regime for the SLy4 interaction resulted in nuclei with larger mass and atomic numbers than those of the SkM interaction.

In this paper, we perform calculations of shear modulus and magneto-elastic mode fre- 
quencies using the SLy4, SkM and Sk272 nucleon-nucleon interactions. Saturation nuclear matter properties of those interactions are listed in Table I. It is evident from the table that those nucleon-nucleon interactions differ in the symmetry energy and its slope coefficient from one interaction to the other. It is to be seen how the behaviour of the symmetry energy and its slope coefficient in the sub-saturation density would impact the compositions of magnetised crusts, its shear modulus and finally magneto-elastic modes.

We calculate the shear modulus using Eq.(4) and the above mentioned models of magnetised crusts. Figure 1 displays the shear modulus as a function of mass density for three nucleon-nucleon interactions of Table I with and without magnetic fields. Here we have shown results for $B_{*}=B / B_{c}=10^{4}$ where $B_{c}=4.414 \times 10^{13} \mathrm{G}$, where $B$ denotes the magnetic field strength at the pole. When the field strength is $<10^{17} \mathrm{G}$, the shear modulus does not show any appreciable change from that of the zero field because of large numbers of Landau levels are populated in this case. As the field strength is increased, less numbers of Landau levels are populated. For $B_{*}=10^{4}$ i.e. $4.414 \times 10^{17} \mathrm{G}$, the shear modulus is enhanced due to the population of all electrons in the zeroth Landau level. In all three cases, the shear modulus increases with mass density well before the crust-core interface. It is observed from Fig. 1 that the shear modulus is highest for the SLy4 nucleon-nucleon interaction whereas it is the lowest for the Sk272 interaction. This can be understood by noting that the symmetry energy at sub-saturation densities is highest for the SLy4 interaction. In this density regime, the symmetry energy decreases from its value at the saturation density according to the slope coefficient $(L)$. As the SLy4 interaction has the lowest value of $L$ (see Table I), it has the highest value of the symmetry energy among all three nucleon-nucleon interactions. Higher symmetry energy leads to higher proton fraction and consequently higher electron fraction due to the charge neutrality. Therefore, higher symmetry energy implies higher shear modulus as is evident from Eq. (4). The shear modulus and shear speed $v_{s}=(\mu / \rho)^{1 / 2}$ are extrapolated to the zero value at the crust-core interface for magnetised as well as non-magnetised crusts. At densities close to the crust-core boundary nuclei can take various non-spherical shapes collectively known as nuclear pasta [53 55]. As the detailed nature of this pasta phase is not fully settled and there is no calculation of the shear modulus of this phase yet and as the shear modulus should vanish at the crust-core boundary, we extrapolate the shear modulus and shear speed $v_{s}=(\mu / \rho)^{1 / 2}$ to the zero value at the crust-core interface for magnetised as well as non-magnetised crusts. This approach 
is similar to that of Ref. [31] where an arbitrary fit was used so that the shear modulus smoothly decreases to zero at the crust-core interface, in the absence of magnetic fields. We generate profiles of the shear modulus as a function of radial distance in a neutron star for calculating frequencies of magneto-elastic modes. The shear modulus profiles along with the profiles of energy density and pressure are obtained by solving the TOV equation. In this context, we construct the EoS of dense nuclear matter in strong magnetic fields in neutron star core using a relativistic mean field model with the GM1 parameter set as described in Ref.[56 58]. This EoS of dense nuclear matter is matched with the EoS of the crust and used in the TOV equation.

\section{A. CME modes}

First we study the magneto-elastic modes confined to the crust only. We investigate the dependence of these mode frequencies on the compositions and the shear modulus of magnetised crusts. Earlier all calculations were performed using non-magnetic neutron star crusts. Here we exploit models of non-magnetic as well as magnetic crusts which were already described in this section. We consider CME modes of a neutron star of mass 1.4 $M_{\odot}$. Frequencies of fundamental $(n=0, \ell=2)$ CME modes are plotted with magnetic fields in Fig. 2 for all three nucleon-nucleon interactions. Here $n$ gives the number of radial nodes in the eigenfunction $\mathcal{Y}(r)$, in the crust. It is observed that in each case the frequency increases very slowly with magnetic field for $B^{*}<100$. But for $B^{*}>100$, the frequency increases linearly with magnetic fields. This behavior was also observed in earlier studies [17, 42]. The frequencies corresponding to the SLy4 interaction for $B^{*}<100$, are almost two times higher than those of the SkM and Sk272 interactions. This is the direct consequence of the higher value of shear modulus for the SLy4 interaction than the other two interactions. However, there are no differences between our results with and without magnetised crusts. This shows that the increase in shear modulus due to magnetic field is too small to change the fundamental modes even for very high fields $\left(\gtrsim 10^{17} \mathrm{G}\right)$.

Figure 3 shows frequencies of CME modes corresponding to $n=0$ plotted as a function of $\ell$ values for a $1.4 M_{\odot}$ neutron star, magnetic field $B_{*}=10^{4}$ and all three nucleonnucleon interactions. Furthermore, we calculate frequencies using the non-magnetic as well as magnetic crusts. In all cases frequency increases with higher $\ell$ values. For higher values 
of $\ell$, frequencies with magnetic crusts are found to be slightly smaller than those of nonmagnetic crusts, i.e. when the effect of magnetic field on the crustal composition is neglected. This is true for all three nucleon-nucleon interactions used in this calculation. The small decrease in frequencies in case of magnetised crusts is due to increase of radius $(R)$ of the star for $B_{*}=10^{4}$ as is evident from Table II because fundamental frequencies are inversely proportional to $R$.

We continue our investigation on frequencies of first overtones $(n=1)$ of CME modes in the presence of magnetic fields. Frequencies of first overtones are shown as a function of $\ell$ values for a neutron star of $1.4 M_{\odot}$, magnetic field $B_{*}=10^{4}$ and all nucleon-nucleon interactions of Table I in Fig. 4. It is observed that the frequencies obtained with magnetised crusts are significantly suppressed compared with those of non-magnetised crusts for each nucleon-nucleon interaction and for all values of $\ell$. This is understood if we remember the fact that the radius of a star is sensitive to the crustal EoS. Since strong magnetic fields $\left(\gtrsim 10^{17} \mathrm{G}\right.$ ) change the composition as well as EoS of the crust, the stellar radius as well as crustal thickness also get affected. In Table [1, we have shown the radius $(R)$ and the ratio of the crust thickness $(\triangle \mathrm{R})$ to the radius of a neutron star for $B *=0$ and $B_{*}=10^{4}$, for all three nuclear interactions. From the table we see that the value of $\triangle R / R$ is larger for $B *=10^{4}$ than for $B=0$. It was noted that the ratio of the crust thickness to the radius of a neutron star was inversely proportional to the frequencies of overtones [17]. Hence, it explains why overtone frequencies are smaller for magnetised crusts with $B *=10^{4}$, even though the shear moduli are little larger for this case than that of $B_{*}=0$. The effects of nucleon-nucleon interactions are evident from the figure where the results of the SkM lie at the top and those of the SLy4 are at the bottom. This can also be understood from Table III if we note that the ratio $(\triangle R / R)$ is the highest for the SLy4 interaction and lowest for the SkM interaction.

The dependence of frequencies of the fundamental mode and higher harmonics on neutron star masses is demonstrated in Fig. 5 for the SLy4, SkM and Sk272 nucleon-nucleon interactions. Here the frequencies corresponding to $n=0$ and $\ell=2,3,4$ are shown as a function of neutron star masses for a magnetic field $B=8 \times 10^{14} \mathrm{G}$. For all cases, frequencies of CME modes decrease with increasing mass, whereas higher $\ell$ values lead to higher frequencies. It is observed from Fig. 5 that frequencies corresponding to (non)magnetic crusts based on the SLy4 nucleon-nucleon interaction are much higher than those of other 
two nucleon-nucleon interactions. When the calculated frequencies are compared with the frequencies of observed QPOs, the latter might put a strong constraint on the EoS if masses of neutron stars are known accurately.

Next, we compare the calculated frequencies of CME modes with frequencies of observed QPOs. These comparisons are shown in Tables [II and IV. Here we have also included QPO of $57 \mathrm{~Hz}$ found recently by Huppenkothen et al. [14] in the short bursts of SGR 1806-20. For SGR1806-20, our results in Table II are obtained using the magnetised crusts of 1.3, 1.4 and $1.7 M_{\odot}$ neutron stars based on the SLy4, SkM and Sk272 nucleon-nucleon interactions, respectively, and magnetic field $B=8 \times 10^{14} \mathrm{G}$. It is noted that calculated frequencies below $93 \mathrm{~Hz}$ for each nucleon-nucleon interaction can not explain the observed frequencies whereas our results above $93 \mathrm{~Hz}$ are in very good agreement with observed QPO frequencies [4, 10, 11]. Similarly for SGR 1900+14, we calculate CME mode frequencies using magnetised crusts of 1.7, 1.2 and $1.2 M_{\odot}$ neutron stars corresponding to the SLy4, SkM and Sk272 nucleon-nucleon interactions and $B=4 \times 10^{14} \mathrm{G}$. These results are shown in Table IV] Our calculated frequencies for all three nucleon-nucleon interactions are in agreement with the observed QPO frequencies of SGR 1900+14 [12].

\section{B. GME modes}

First we calculate pure Alfvén modes of a neutron star of mass $1.4 M_{\odot}$, by ignoring the presence of the solid crust. In Fig. 6, we show the pure Alfvén mode corresponding to $n=0 ; \ell=2$ as a function of magnetic field $\left(B_{*}\right)$. Here, $n$ stands for the number of radial nodes in the eigenfunctions, in the liquid core. We see that the frequency of this mode increases linearly with magnetic field and become equal to those of the CME modes above $B_{*}=100$. Next, we calculate corresponding GME mode frequencies for various magnetic fields, taking magnetic crusts into consideration. Magnetised crusts used here are calculated with the SLy4, SkM and Sk272 nucleon-nucleon interactions. It is observed that at low magnetic fields global mode frequencies have higher values as compared to those of pure Alfvén modes. The GME modes are found to be confined to the core for low magnetic field strengths. This scenario is similar to the reflection of GME modes at the crust-core interface as manifested in the state-of-the-art model of Gabler et al. [29]. Consequently, this

leads to higher frequencies for GME modes compared with those of pure Alfvén modes. But 
at higher magnetic fields, GME mode frequencies merge with that of pure Alfvén modes. This happens because at higher values of fields $\left(B \geq 4.14 \times 10^{15}\right)$ shear modulus becomes negligible as compared to the magnetic effect $\left(\mu \ll B^{2}\right)$; in other words Alfvén velocity $(B / \sqrt{4 \pi \rho})$ becomes much larger compared to the shear velocity $(\sqrt{\mu / \rho})$. We also show the frequencies of CME modes for comparison. It is also evident from Fig. 6 that the effects of crusts on frequencies disappear at very high magnetic fields $B_{*}>100$ and oscillations become magnetic oscillations [59].

To see the effects of magnetic crusts on GME modes we calculate these modes with and without magnetic crusts based on the SLy4, SkM and Sk272 nucleon-nucleon interactions. Figures 7 and 8 show results for modes with $n=0$ and $n=1$, respectively as a function of $\ell$ for a neutron star of mass $1.4 M_{\odot}$ and magnetic field $B_{*}=10^{4}$. We can see there is no significant change in frequencies if the crust is considered to be magnetic. For fundamental modes in Fig. 7, there is no appreciable change in frequencies with and without magnetic crusts. Unlike Fig. 3 for CME modes, GME modes are insensitive to the small change in R. In case of first overtones in Fig. 8, we do not find any appreciable effects of crusts on frequencies because the magnetic field $B_{*}=10^{4}$ is so high that oscillations become magnetic oscillations.

We also attempt to match the observed frequencies with those of calculated GME modes. The results are shown in Tables V and VI. For SGR1806-20, we compute frequencies using the magnetised crusts of 1.5, 1.4 and $1.4 M_{\odot}$ neutron stars based on the SLy4, SkM and Sk272 nucleon-nucleon interactions, respectively, and magnetic field $B=3.1 \times 10^{15} \mathrm{G}$. These results are given by Table $\mathrm{V}$. We find that the calculated frequencies agree well with the lower and higher frequencies of observed QPOs. However, it is noted that large values of $n$ are needed to fit higher frequencies. This feature for higher frequencies was also obtained by Sotani et al. [42]. On the other hand, we exploit magnetised crusts of 1.4, 1.3 and $1.3 \mathrm{M}_{\odot}$ neutron stars corresponding to the SLy4, SkM and Sk272 nucleon-nucleon interactions and magnetic field $B=1.34 \times 10^{15} \mathrm{G}$ for SGR $1900+14$. The Table VI demonstrates that the calculated frequencies are in good agreement with the observed frequencies of SGR 1900+14. We do not find any appreciable effects of nucleon-nucleon interactions in either table. 


\section{SUMMARY AND CONCLUSIONS}

We have estimated frequencies of global magneto-elastic modes as well as magneto-elastic modes confined to the crust only of magnetars assuming a dipole magnetic field configuration. Frequencies are computed using our models of magnetised crusts based on the SLy4, SkM and Sk272 nucleon-nucleon interactions. Though the formalism used in Sotani et al. [42] and in this calculation are same, magnetised crusts are employed for the first time here. The shear modulus of magnetised crusts is found to be enhanced in strong magnetic fields $\sim 4.414 \times 10^{17}$ $\mathrm{G}$ because electrons populate the zeroth Landau level. It is observed that frequencies of the fundamental $(n=0, \ell=2$ ) CME mode are not sensitive to this enhancement in the shear modulus in strong magnetic fields. On the other hand, frequencies of first overtones $(n=1)$ of CME modes in the presence of strongly quantising magnetic fields are distinctly different from those of the field free case. It is shown that that this is related to the the ratio of the crust thickness to the radius of a magnetar. We have found that at $B_{*}=10^{4}$, the $\triangle R / R$ is increased by $2-4 \%$, which causes frequencies of overtones to decrease by $5-7 \%$,

for the models we used here. For GME modes, the effects of crusts disappear above a critical field $\left(B>4 \times 10^{15} \mathrm{G}\right)$ and oscillations become magnetic oscillations. We have compared frequencies of CME and GME modes calculated using different stellar models, magnetic field strengths and magnetised crusts based on three nucleon-nucleon interactions with frequencies of observed QPOs and conclude that the agreement is reasonable for SGR 1900+14 in both cases. However, the calculated frequencies of CME modes do not match with lower frequencies of SGR 1806-20, but can explain higher frequencies well. In the case of GME modes, we find the opposite trends in fitting the frequencies of SGR 1806-20. Finally new results that we have obtained would be reproduced even in a sophisticated MHD calculation.

[1] S.A. Olaunsen and V.M. Kaspi, Astrophys. J. Suppl. Ser., 212, 6 (2014).

[2] K. Hurley et al., Nature 397, L41 (1999).

[3] C. Barat et al., Astron. Astrophys. 126, 400 (1983).

[4] G.L. Israel et al, ApJ 628, L53 (2005).

[5] A.L. Watts, in Neutron Star Crust, edited by Bertulani C. A., and Piekarewicz J., (Nova 
Science, NY, 2012); arXiv:1111.0514.

[6] A.L. Watts and T.E. Strohmayer, Adv. Sp. Res. 40, 1446 (2007).

[7] R.C. Duncan and C. Thompson, ApJ 392, L9 (1992).

[8] R.C. Duncan, ApJ 498, L45 (1998).

[9] C. Kouveliotou C., et al., Nature 393, L235 (1998).

[10] A.L. Watts and T.E. Strohmayer, ApJ 637, L117 (2006).

[11] T.E. Strohmayer and A.L. Watts, ApJ 653, 593 (2006).

[12] T.E. Strohmayer and A.L. Watts, ApJ 632, L111 (2005).

[13] D. Huppenkothen et al, ApJ 787, 128 (2014).

[14] D. Huppenkothen, L. M. Heil, A.L. Watts and E. Göğös, ApJ 795, 114 (2014).

[15] D. Huppenkothen, A.L. Watts and Y. Levin, ApJ 793, 129 (2014)

[16] A.L. Piro, ApJ 634, L153 (2005).

[17] H. Sotani, K.D. Kokkotas and N. Stergioulas, MNRAS 375, 261 (2007).

[18] L. Samuelsson and N. Andersson, MNRAS 374, 256 (2007).

[19] H. Sotani, K.D. Kokkotas and N. Stergioulas, MNRAS 385, L5 (2008).

[20] H. Sotani, A. Colaiuda and K.D. Kokkotas, MNRAS 385, 2161 (2008).

[21] A.W. Steiner and A.L. Watts, Phys. Rev. Lett. 103, 181101 (2009).

[22] Y. Levin, MNRAS 377, 159 (2007).

[23] A. Colaiuda, H. Beyer and K.D. Kokkotas, MNRAS 396, 1441 (2009).

[24] P. Cerdá-Durán, N. Stergioulas and J.A. Font., MNRAS 397, 1607 (2009)

[25] Y. Levin, MNRAS 368, L35 (2006)

[26] M. van Hoven and Y. Levin, MNRAS 410, 1036 (2011).

[27] M. van Hoven and Y. Levin, MNRAS 420, 3035 (2012).

[28] A. Colaiuda and K.D. Kokkotas, MNRAS 414, 3014 (2011).

[29] M. Gabler, P. Cerdá-Durán, N. Stergioulas, J.A. Font and E. Müller, MNRAS 421, 2054 (2012).

[30] M. Gabler, P. Cerdá-Durán, J.A. Font, E. Müller and N. Stergioulas, MNRAS 430, 1811 (2013).

[31] H. Sotani, MNRAS 417, L70 (2011).

[32] N. Andersson, K Glampedakis and L. Samuelsson, MNRAS 396, 894 (2009).

[33] A. Passamonti and S. K. Lander, MNRAS 438, 156 (2013). 
[34] M. Gabler, P. Cerdá-Durán, N. Stergioulas, J.A. Font and E. Müller, PRL 111, 211102 (2013).

[35] M. Sinha and A. Sedrakian, PRC 91, 035805 (2015).

[36] R. Nandi and D. Bandyopadhyay, J. Phys. Conf. Ser. 312, 042016 (2011).

[37] R. Nandi, D. Bandyopadhyay, I.N. Mishustin and W. Greiner, ApJ 736, 156 (2011).

[38] B.W. Carrol et al., ApJ 305, 767 (1986).

[39] P.N. McDermott, H.M. van Horn, C. J. Hansen, ApJ 325, 725 (1988).

[40] B.L. Schumaker and K.S. Thorne, MNRAS 203, 457 (1983).

[41] N. Messios, D.B. Papadopolous, N. Stergioulas, MNRAS 328, 1161 (2001).

[42] H. Sotani, K. D. Kokkotas, N. Stergioulas, and M. Vavoulidis, arXiv:astro-ph/0611666

[43] M. Bocquet, S. Bonazzola, E. Gourgoulhon and J. Novak, Astro. Astrophys. 301, 757 (1995)

[44] D. Chatterjee, T. Elghozi, J. Novak and M. Oertel, MNRAS 447, 3785 (2015).

[45] P.N. McDermott,H.M. van Horn, J.F. Scholl, ApJ 268, 837 (1983).

[46] S. Ogata and S. Ichimaru, Phys. Rev. A 42, 4867 (1990).

[47] T. Strohmayer, H.M. van Horn, S. Ogata, H. Iyetomi and S. Ichimaru, ApJ 375, 679 (1991).

[48] P. Haensel, in Lecture Notes in Physics: Physics of Neutron Star Interiors, edited by Blaschke D., Glendenning N.K. and Sedrakian A., 578, 127 (Springer, Heidelberg, 2001).

[49] G. Baym, C. Pethick and P. Sutherland, ApJ 170, 299 (1971)

[50] P. Bonche, S. Levit and D. Vautherin, Nucl. Phys. A427, 278 (1984).

[51] P. Bonche, S. Levit and D. Vautherin, Nucl. Phys. A436, 265 (1985).

[52] T. Sil, J.N. De, S.K. Samaddar, X. Vinas, M. Centelles, B.K. Agrawal and S.K. Patra, Phys. Rev. C 66, 045803 (2002).

[53] D. G. Ravenhall, C. J. Pethick and J. R. Wilson Phys. Rev. Lett. 50, 2066 (1983)

[54] M. Hashimoto, H. Seki and M. Yamada, Prog. Theor. Phys. 71320 (1984)

[55] R. Nandi and S. Schramm, arXiv:nucl-th/1601.01842.

[56] S. Chakrabarty, D. Bandyopadhyay and S. Pal, Phys. Rev. Lett. 78, 2898 (1997).

[57] M. Sinha and D. Bandyopadhyay, Phys. Rev. D 79, 123001 (2009).

[58] N.K. Glendenning and S.A. Moszkowski, Phys. Rev. Lett. 67, 2414 (1991).

[59] H. Sotani, Phys. Rev. D 92, 104024 (2015). 
TABLE I: Saturation nuclear matter properties of different Skyrme nucleon-nucleon interactions used in this work such as saturation density $\left(\rho_{0}\right)$, binding energy $(\mathrm{BE})$, incompressibility $(\mathrm{K})$, symmetry energy $(\mathrm{J})$ and its slope coefficient $(\mathrm{L})$

\begin{tabular}{cccccc}
\hline Parameter set & $\rho_{0}$ & $\mathrm{BE}$ & $\mathrm{K}$ & $\mathrm{J}$ & $\mathrm{L}$ \\
& $\left(\mathrm{fm}^{-3}\right)$ & $(\mathrm{MeV})$ & $(\mathrm{MeV})$ & $(\mathrm{MeV})$ & $(\mathrm{MeV})$ \\
\hline SLy4 & 0.16 & 15.97 & 229.91 & 32.00 & 45.94 \\
SkM & 0.16 & 15.77 & 216.61 & 30.75 & 49.34 \\
Sk272 & 0.155 & 16.28 & 271.51 & 37.40 & 91.67 \\
\hline
\end{tabular}

TABLE II: Radius and crust thickness for all three interactions at $B *=0$ and $B *=10^{4}$

\begin{tabular}{lcc||cc}
\hline \multicolumn{3}{c}{$B=0$} & \multicolumn{2}{c}{$B_{*}=10^{4}$} \\
Set & $\mathrm{R}(\mathrm{km})$ & $\Delta \mathrm{R} / \mathrm{R}$ & $\mathrm{R}(\mathrm{km})$ & $\triangle \mathrm{R} / \mathrm{R}$ \\
\hline SLy4 & 13.972 & 0.096 & 13.987 & 0.100 \\
SkM & 13.875 & 0.086 & 13.892 & 0.088 \\
Sk272 & 13.910 & 0.089 & 13.927 & 0.092 \\
\hline
\end{tabular}


TABLE III: Frequencies of CME modes calculated using magnetised crusts based on the SLy4, SkM and Sk272 nucleon-nucleon interactions are compared with observed QPO frequencies of SGR 180620 4, 10, 11, 14]. The magnetic field used in this calculation is $B=8 \times 10^{14}$ G. Here $f, n$ and $\ell$ represent frequency, radial node and angular node, respectively

\begin{tabular}{ccccccccccc}
\hline Observed & \multicolumn{8}{c}{ Calculated frequency $(\mathrm{Hz})$} \\
frequency $(\mathrm{Hz})$ & \multicolumn{1}{c}{ SLy4 } & \multicolumn{2}{c}{ SkM } & \multicolumn{2}{c}{ Sk272 } \\
& $\left(1.3 M_{\odot}\right)$ & & $\left(1.4 M_{\odot}\right)$ & & $\left(1.7 M_{\odot}\right)$ \\
\hline & $f$ & $\mathrm{n}$ & $\ell$ & $f$ & $\mathrm{n}$ & $\ell$ & $f$ & $\mathrm{n}$ & $\ell$ \\
18 & 20.0 & 0 & 2 & 13.0 & 0 & 2 & 18.0 & 0 & 3 \\
26 & - & - & - & 20.7 & 0 & 3 & 24.3 & 0 & 4 \\
30 & 31.7 & 0 & 3 & 27.8 & 0 & 4 & 30.3 & 0 & 5 \\
57 & 53.1 & 0 & 5 & 55.0 & 0 & 8 & 59.5 & 0 & 10 \\
92.5 & 94.1 & 0 & 9 & 94.5 & 0 & 14 & 93.9 & 0 & 16 \\
150 & 154.6 & 0 & 15 & 152.6 & 0 & 23 & 150.0 & 0 & 26 \\
626 & 627.9 & 1 & 16 & 626.9 & 1 & 27 & 625.9 & 1 & 34 \\
1838 & 1834.5 & 4 & 2 & 1836.3 & 4 & 2 & 1841.9 & 4 & 2 \\
\hline
\end{tabular}


TABLE IV: Same as Table III but for SGR 1900+14 [12]. The magnetic field used in this calculation is $B=4 \times 10^{14} \mathrm{G}$.

\begin{tabular}{cccccccccc}
\hline Observed & \multicolumn{6}{c}{ Calculated frequency $(\mathrm{Hz})$} \\
frequency $(\mathrm{Hz})$ & SLy4 & \multicolumn{2}{c}{ SkM } & \multicolumn{2}{c}{ Sk272 } \\
& $\left(1.7 M_{\odot}\right)$ & $\left(1.2 M_{\odot}\right)$ & \multicolumn{2}{c}{$\left(1.2 M_{\odot}\right)$} \\
\hline & $f$ & $\mathrm{n}$ & $\ell$ & $f$ & $\mathrm{n}$ & $\ell$ & $f$ & $\mathrm{n}$ & $\ell$ \\
28 & 28.4 & 0 & 3 & 28.3 & 0 & 4 & 26.6 & 0 & 4 \\
54 & 56.7 & 0 & 6 & 55.8 & 0 & 8 & 52.5 & 0 & 8 \\
84 & 84.2 & 0 & 9 & 82.7 & 0 & 12 & 83.8 & 0 & 13 \\
155 & 156.4 & 0 & 17 & 155.2 & 0 & 23 & 157.1 & 0 & 25 \\
\hline
\end{tabular}

TABLE V: GME mode frequencies obtained using the magnetised crusts based on the SLy4, SkM and Sk272 nucleon-nucleon interactions are compared with observed frequencies in SGR 1806-20. The magnetic field used in this calculation is $B=3.1 \times 10^{15} \mathrm{G}$.

\begin{tabular}{cccccccccccc}
\hline Observed & \multicolumn{6}{c}{ Calculated frequency $(\mathrm{Hz})$} \\
frequency $(\mathrm{Hz})$ & \multicolumn{1}{c}{ SLy4 } & \multicolumn{4}{c}{ SkM } & \multicolumn{2}{c}{ Sk272 } \\
& $\left(1.5 M_{\odot}\right)$ & \multicolumn{2}{c}{$\left(1.4 M_{\odot}\right)$} & \multicolumn{2}{c}{$\left(1.4 M_{\odot}\right)$} \\
\hline & $f$ & $\mathrm{n}$ & $\ell$ & $f$ & $\mathrm{n}$ & $\ell$ & $f$ & $\mathrm{n}$ & $\ell$ \\
18 & 17.8 & 0 & 3 & 18.2 & 0 & 3 & 18.1 & 0 & 3 \\
26 & 26.1 & 0 & 6 & 26.1 & 0 & 6 & 25.8 & 0 & 6 \\
30 & 30.7 & 0 & 8 & 30.7 & 0 & 8 & 30.4 & 0 & 8 \\
57 & 57.8 & 1 & 7 & 57.1 & 1 & 6 & 56.7 & 1 & 6 \\
92.5 & 93.0 & 4 & 2 & 91.6 & 2 & 8 & 94.5 & 2 & 9 \\
150 & 150.0 & 6 & 4 & 150.9 & 4 & 10 & 150.3 & 6 & 3 \\
626 & 624.3 & 30 & 6 & 626.4 & 28 & 6 & 628.6 & 27 & 9 \\
1838 & 1837.3 & 96 & 5 & 1836.4 & 97 & 2 & 1835.8 & 87 & 10 \\
\hline
\end{tabular}


TABLE VI: Same as Table V but for the SGR 1900+14. The magnetic field adopted here is $B=1.34 \times 10^{15} \mathrm{G}$.

\begin{tabular}{ccccccccccc}
\hline Observed & \multicolumn{6}{c}{ Calculated frequency $(\mathrm{Hz})$} \\
frequency $(\mathrm{Hz})$ & SLy4 & \multicolumn{2}{c}{ SkM } & \multicolumn{2}{c}{ Sk272 } \\
& $\left(1.4 M_{\odot}\right)$ & $\left(1.3 M_{\odot}\right)$ & \multicolumn{2}{c}{$\left(1.3 M_{\odot}\right)$} \\
\hline & $f$ & $\mathrm{n}$ & $\ell$ & $f$ & $\mathrm{n}$ & $\ell$ & $f$ & $\mathrm{n}$ & $\ell$ \\
28 & 28.0 & 1 & 6 & 28.4 & 1 & 6 & 28.0 & 1 & 5 \\
54 & 54.7 & 3 & 8 & 54.7 & 3 & 7 & 53.6 & 2 & 11 \\
84 & 84.7 & 7 & 5 & 84.1 & 6 & 6 & 84.4 & 5 & 8 \\
155 & 155.6 & 16 & 3 & 154.7 & 14 & 4 & 154.5 & 11 & 9 \\
\hline
\end{tabular}




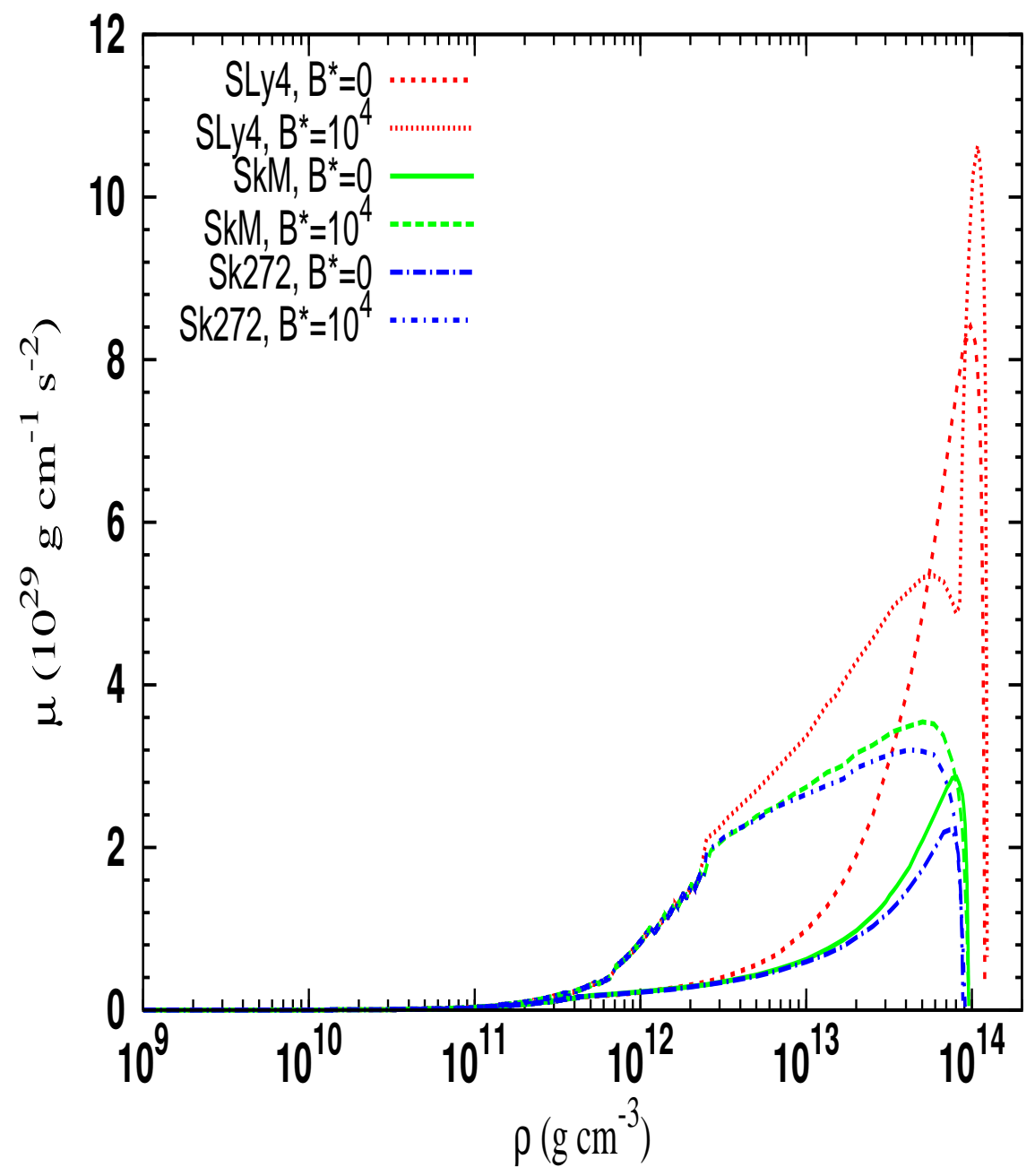

FIG. 1. Shear modulus as a function of mass density for a neutron star of $1.4 M_{\odot}$ with magnetic fields $B_{*}=0$ and $B_{*}=10^{4}$ and Skyrme nucleon-nucleon interactions of Table I. 


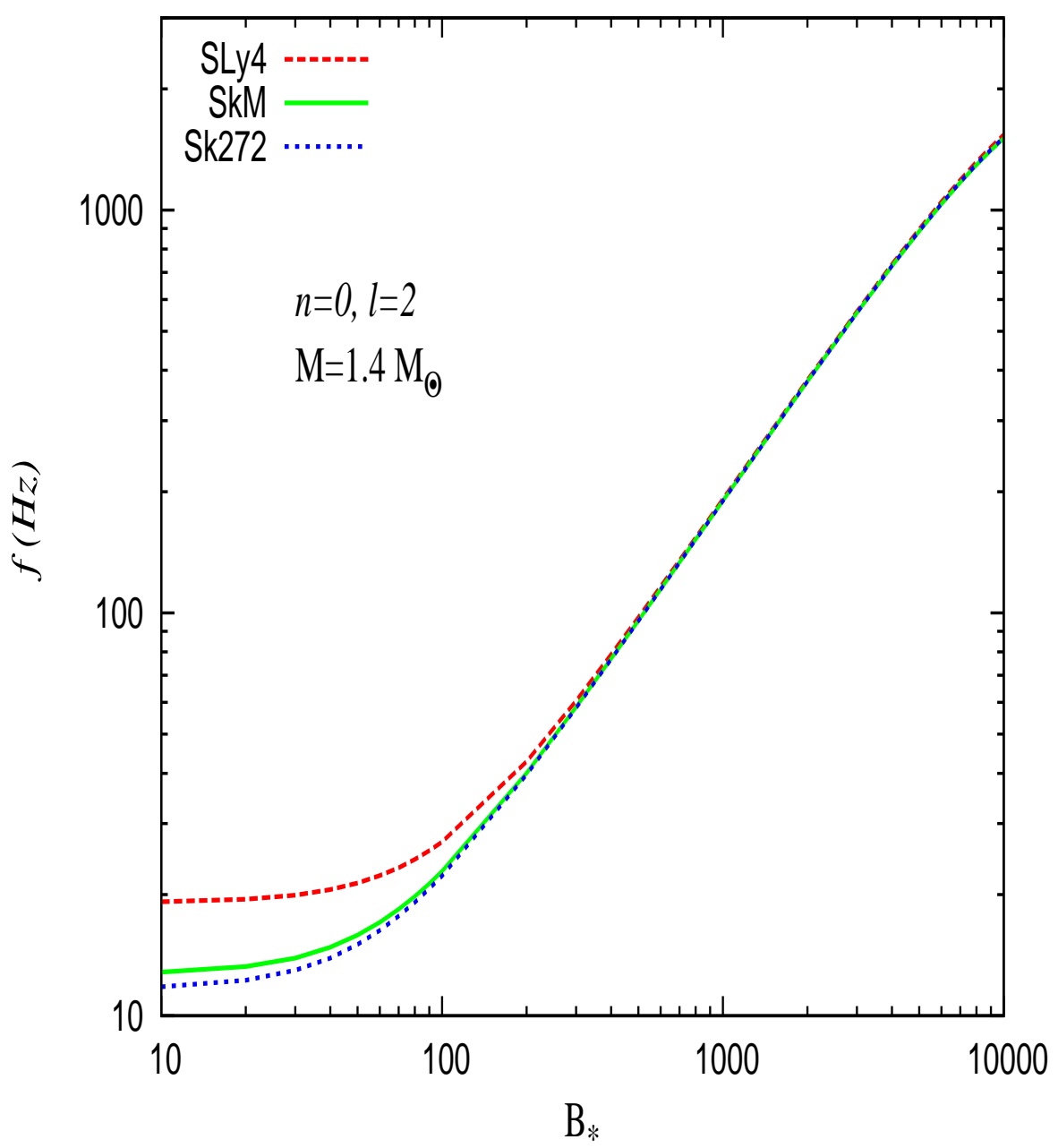

FIG. 2. Frequency of fundamental $(n=0, \ell=2)$ CME mode for a neutron star of $1.4 M_{\odot}$ is shown as a function of magnetic field $B_{*}=B / B_{c}$ where $B_{c}=4.414 \times 10^{13} \mathrm{G}$. Results of our calculations using the SLy4, SkM and Sk272 nucleon-nucleon interactions are shown here. 


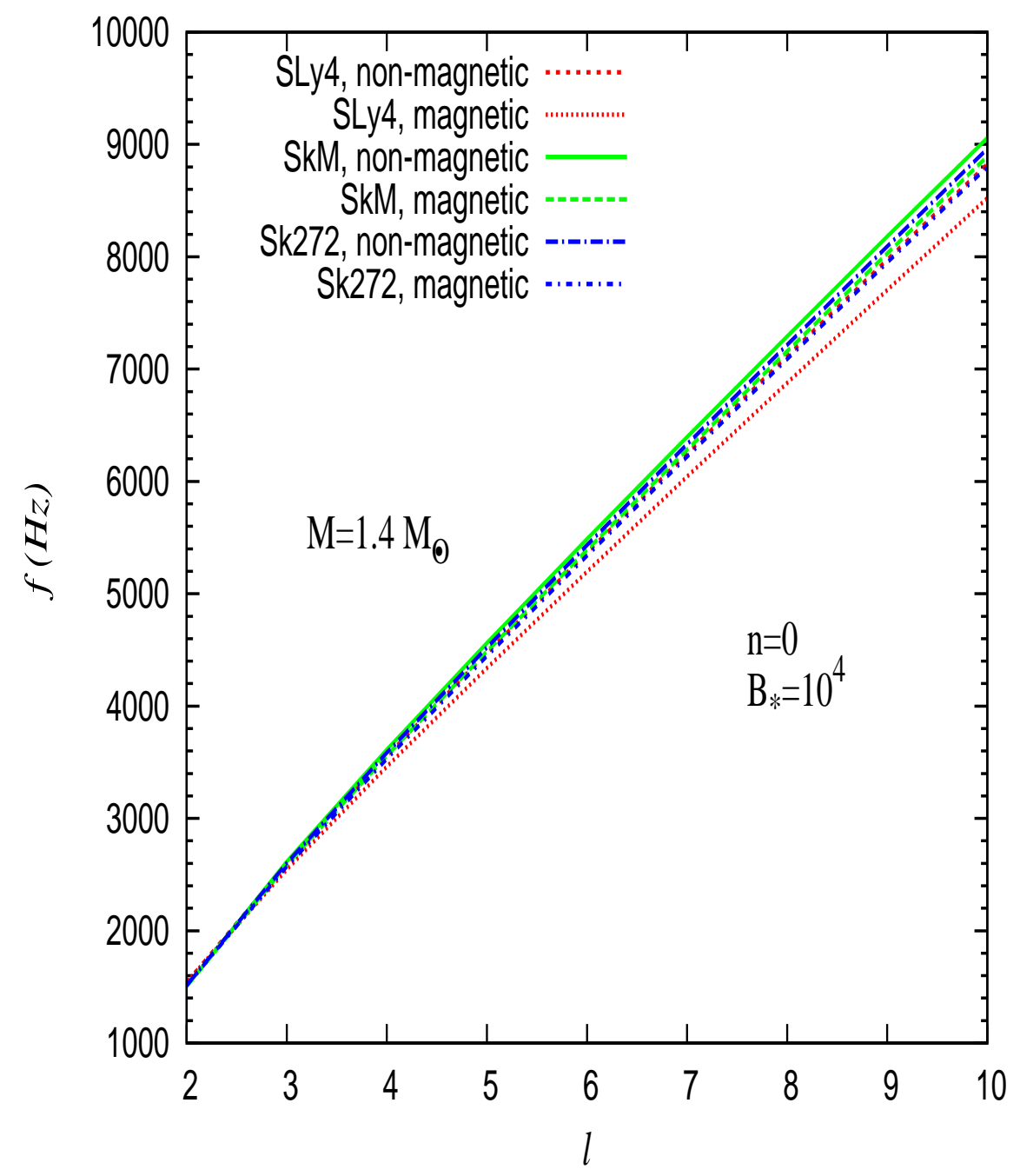

Fig. 3. Fundamental frequencies $(n=0)$ of CME modes are plotted as a function of $\ell$ values with and without magnetic crusts of a $1.4 M_{\odot}$ neutron star based on the SLy4, SkM and Sk272 nucleon-nucleon interactions for $B_{*}=10^{4}$. 


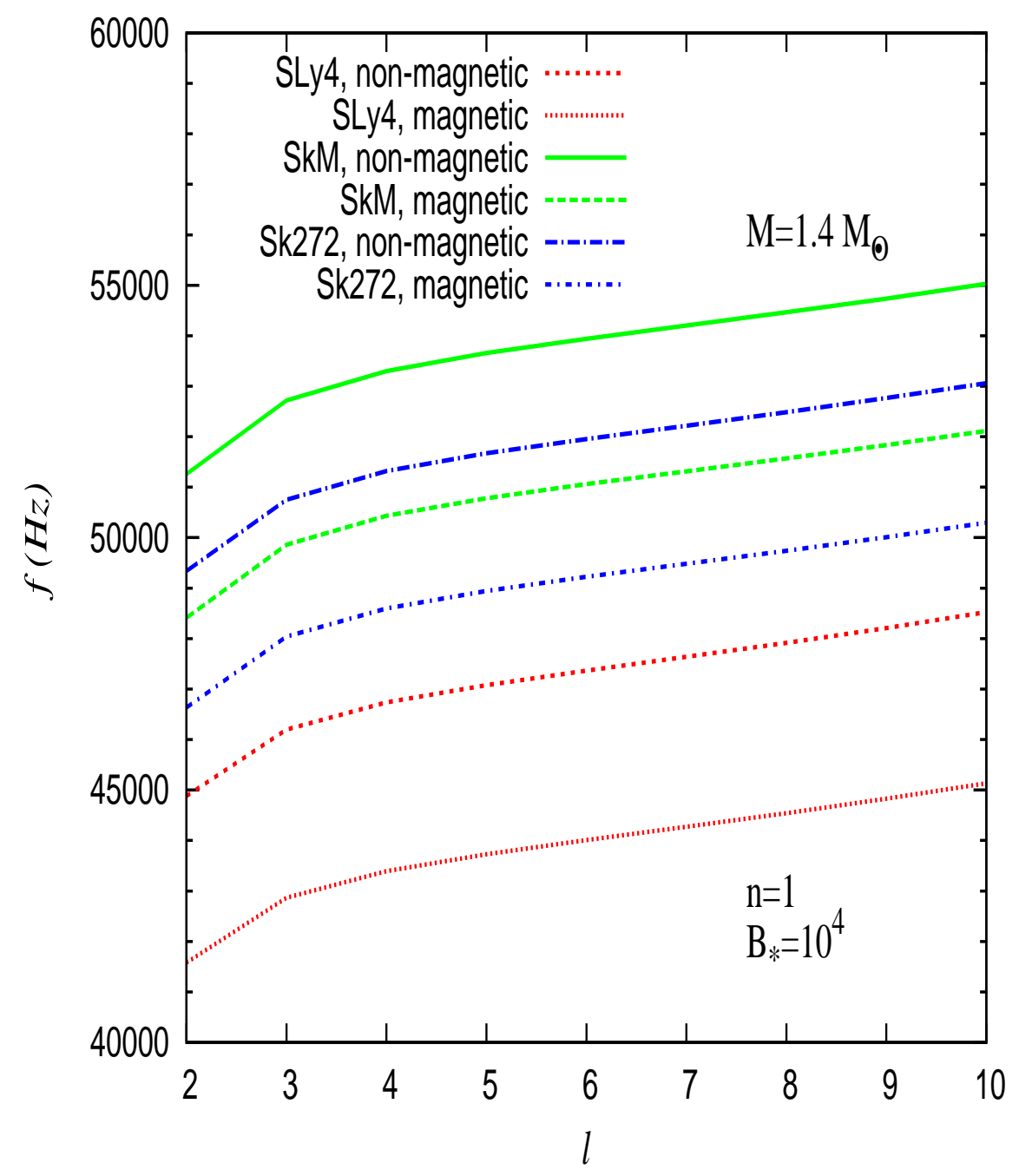

Fig. 4. Frequencies of first overtones $(n=1)$ of CME modes are shown as a function of $\ell$ values with and without magnetic crusts of a $1.4 M_{\odot}$ neutron star based on the SLy4, SkM and Sk272 nucleon-nucleon interactions for $B_{*}=10^{4}$. 


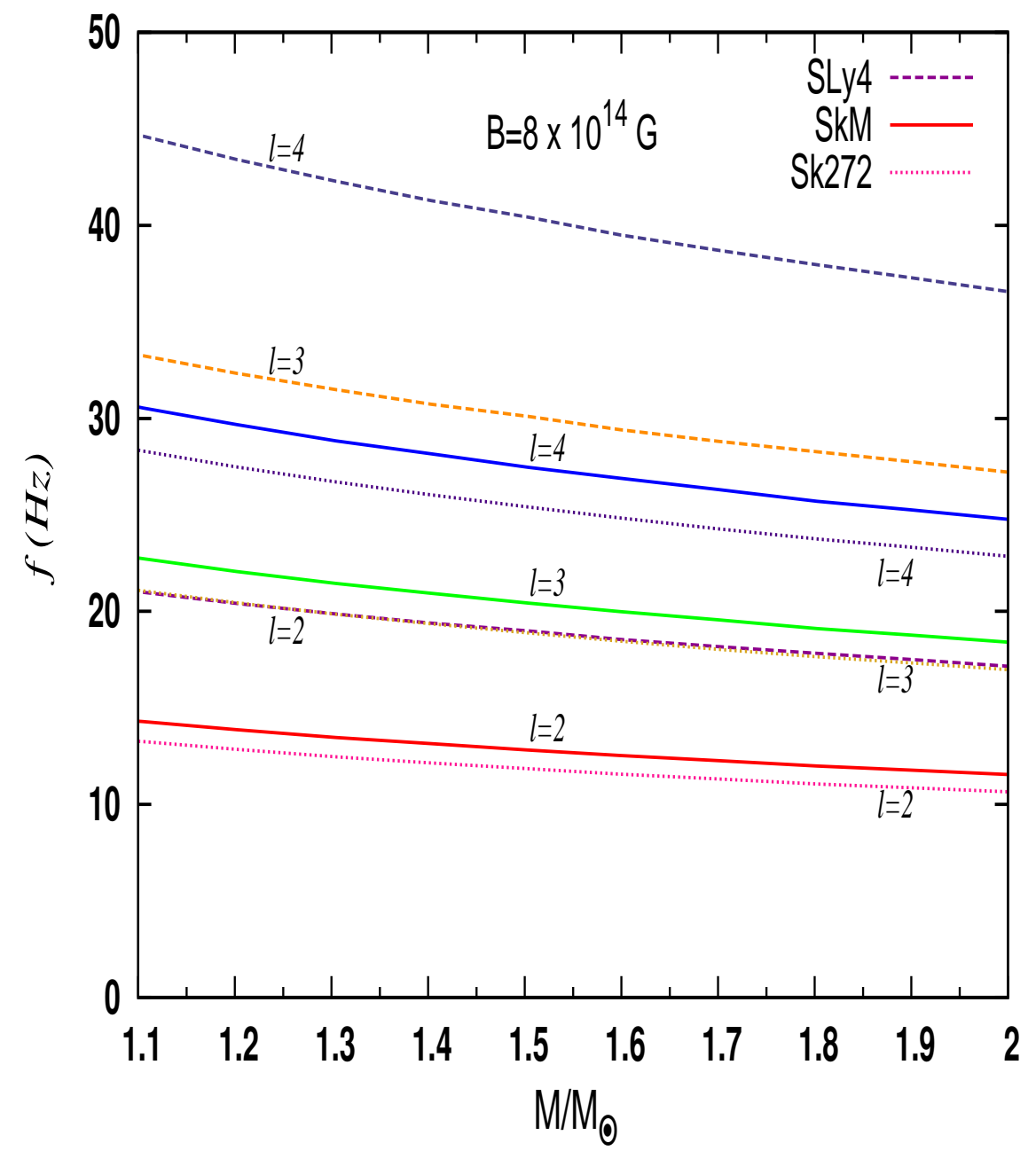

Fig. 5. Frequencies of CME modes corresponding to $n=0$ and $\ell=2,3,4$ are plotted as a function of neutron star mass for a magnetic field $B=8 \times 10^{14} \mathrm{G}$ using magnetised crusts based on the SLy4, SkM and Sk272 nucleon-nucleon interactions. 


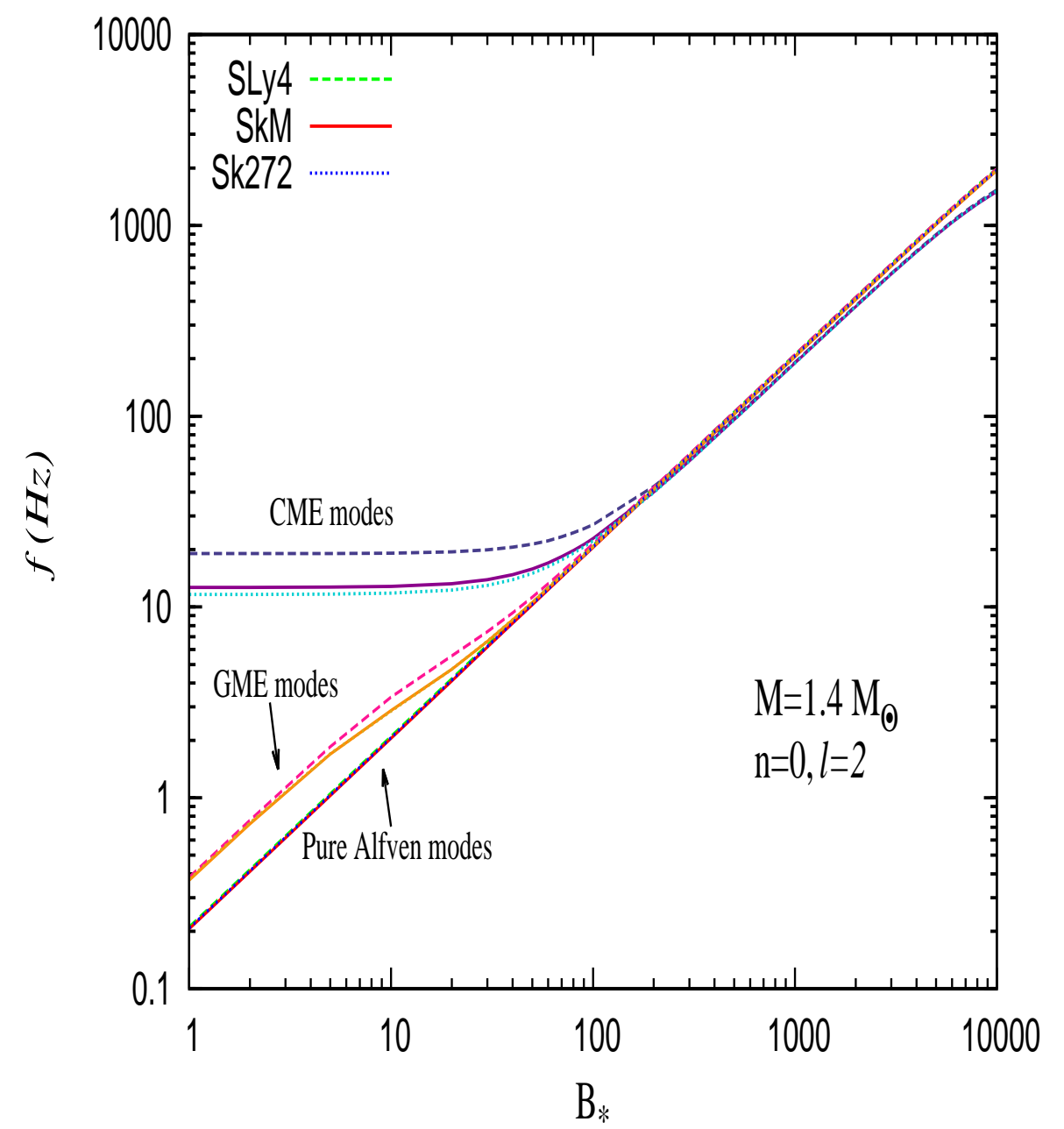

Fig. 6. Comparison of the GME frequencies with pure Alfvén frequencies as well as CME frequencies is shown as a function of magnetic field using the magnetised crusts based on the SLy4, SkM and Sk272 nucleon-nucleon interactions. 


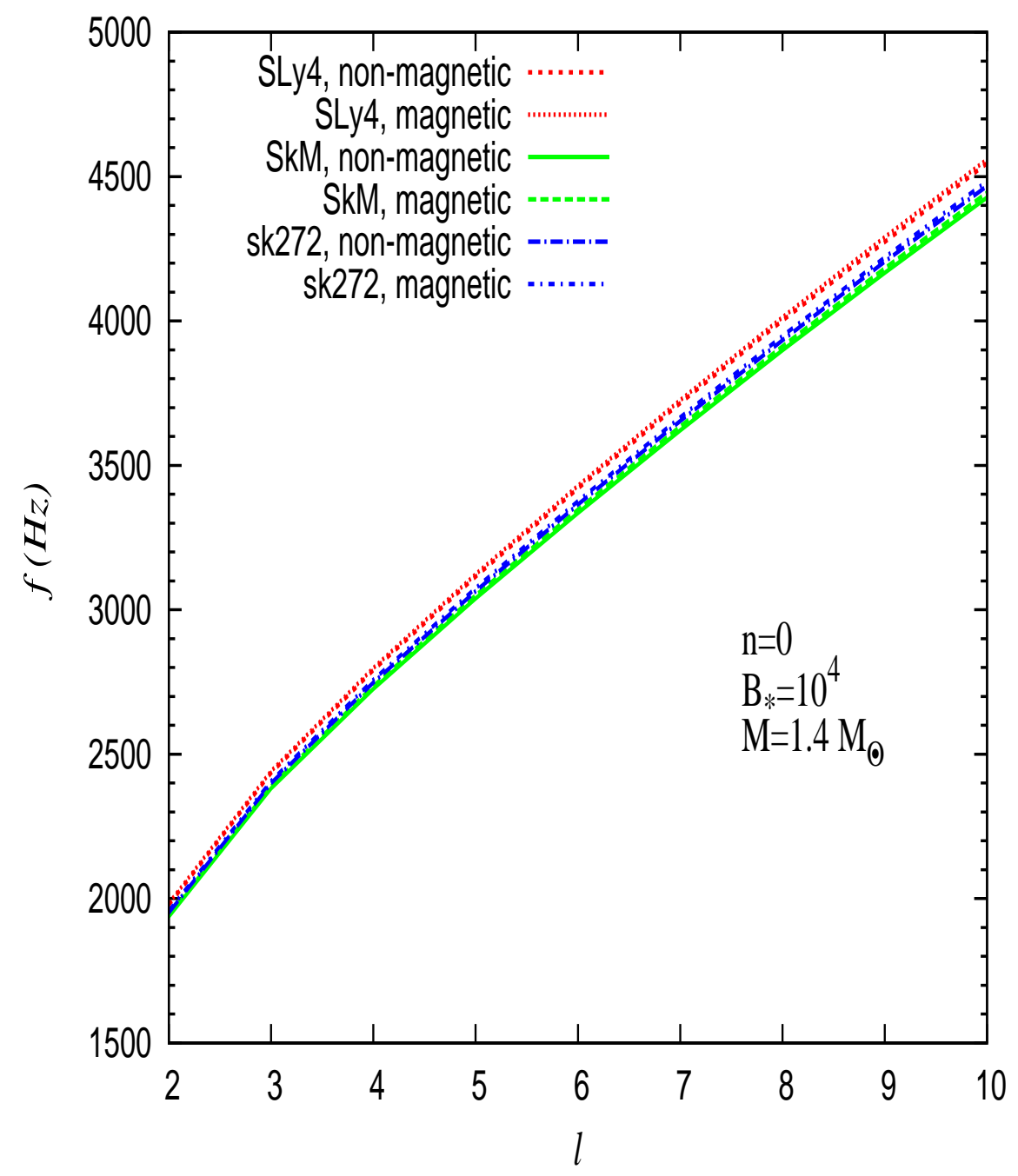

Fig. 7. GME mode frequencies for $n=0$ are shown as a function of $\ell$ values with and without magnetic crusts of a neutron star of mass $1.4 M_{\odot}$ based on the SLy4, SkM and Sk272 nucleonnucleon interactions for $B_{*}=10^{4}$. 


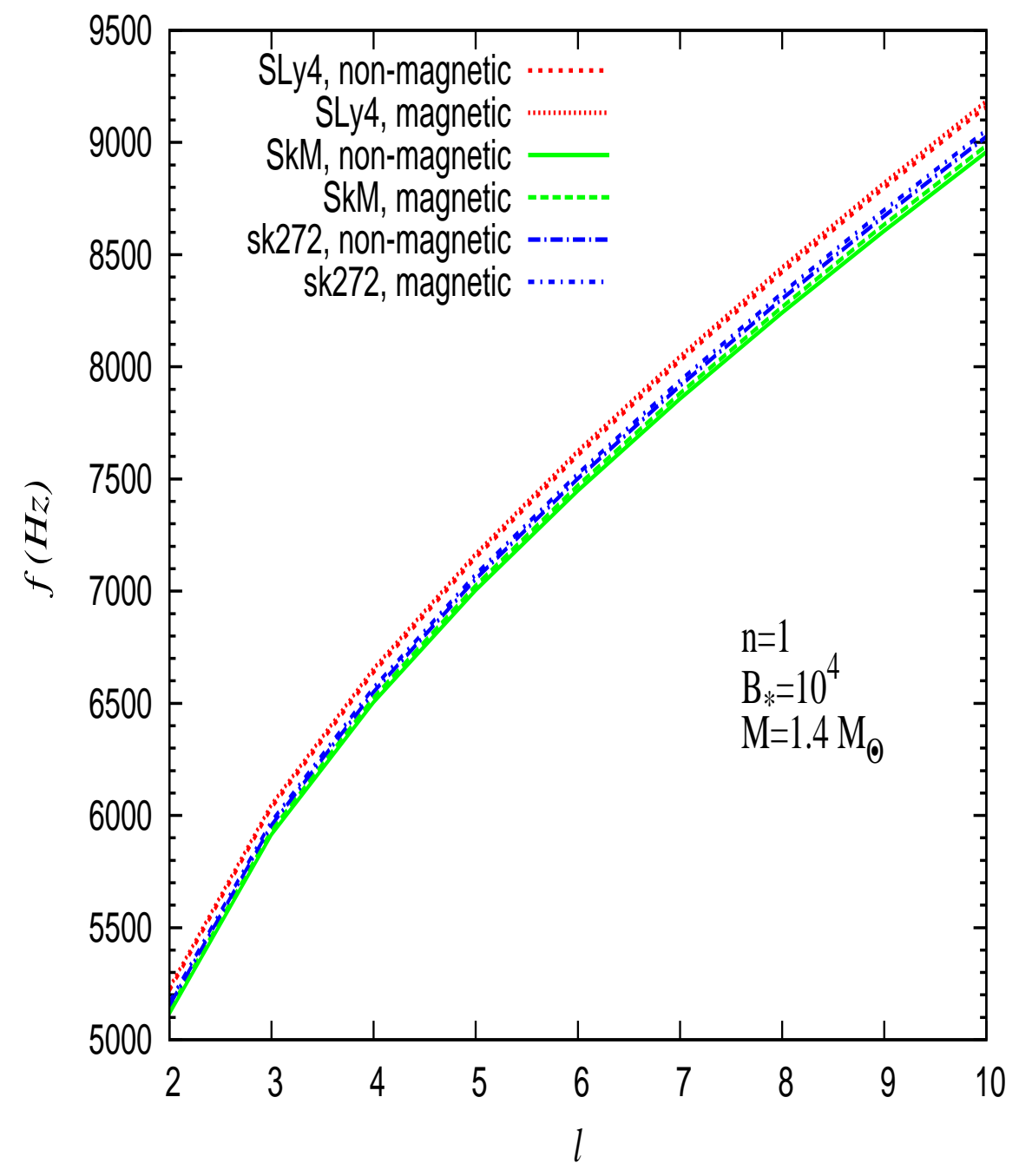

Fig. 8. GME mode frequencies for $n=1$ are plotted as a function of $\ell$ values with and without magnetic crusts of a neutron star of mass $1.4 M_{\odot}$ based on the SLy4, SkM and Sk272 nucleonnucleon interactions for $B_{*}=10^{4}$. 